\title{
Processing System for an Enhanced Vision System
}

\author{
Dennis J. Yelton ${ }^{*}$, Ken L. Bernier ${ }^{\dagger}$ John N. Sanders-Reed ${ }^{*}$
}

\begin{abstract}
Enhanced Vision Systems (EVS) combines imagery from multiple sensors, possibly running at different frame rates and pixel counts, on to a display. In the case of a Helmet Mounted Display (HMD), the user line of sight is continuously changing with the result that the sensor pixels rendered on the display are changing in real time. In an EVS, the various sensors provide overlapping fields of view which requires stitching imagery together to provide a seamless mosaic to the user. Further, different modality sensors may be present requiring the fusion of imagery from the sensors. All of this takes place in a dynamic flight environment where the aircraft (with fixed mounted sensors) is changing position and orientation while the users are independently changing their lines of sight. In order to provide well registered, seamless imagery, very low throughput latencies are required, while dealing with huge volumes of data. This provides both algorithmic and processing challenges which must be overcome to provide a suitable system. This paper discusses system architecture, efficient stitching and fusing algorithms, and hardware implementation issues.
\end{abstract}

Keywords: Enhanced Vision System, multi-sensor, image stitching, image fusion, real-time, helmet mounted display, head-up display, head-down display

\section{INTRODUCTION}

An Enhanced Vision System (EVS) combines imagery from multiple sensors, providing an integrated situational awareness display to the user (figure 1). Depending upon the available system components, this may require stitching imagery from sensors having different, perhaps partially overlapping Fields Of View (FOVs), fusing imagery from different modality sensors having a common FOV, and combining this sensor imagery with synthetic imagery (3D terrain from digital elevation maps, overhead satellite imagery, flight path symbology). The output of an EVS is then presented on a head-down, head-up, or helmet mounted display [1].

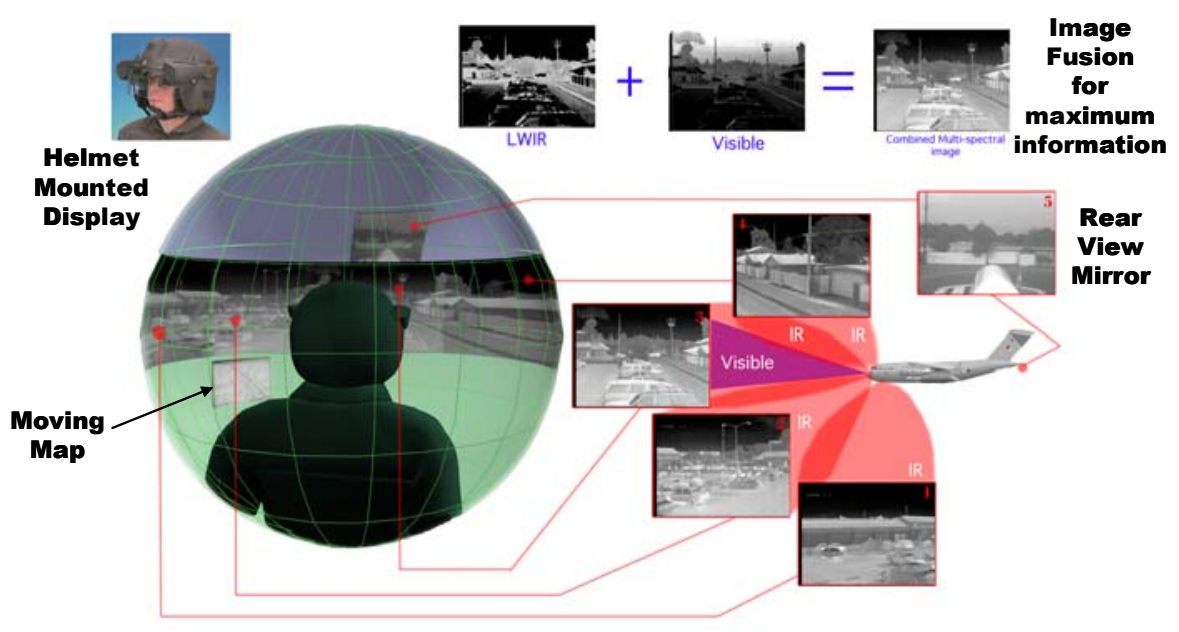

Figure 1. Enhanced Vision System

\footnotetext{
*Boeing-SVS, Inc. 4411 The 25 Way NE, Albuquerque, NM 87109

${ }^{\dagger}$ The Boeing Company, PO Box 516, MC S064-2374, St. Louis, MO 63166
} 
In the case of a Head-Up Display (HUD) or Helmet Mounted Display (HMD), the EVS imagery must be conformal, i.e. properly overlaid on and registered with the external world as viewed by the user. In the case of a HUD or HMD this imposes a strict limit on latency with head motion of the user. For a Head Down Display (HDD), although conformal imagery is neither required nor desired, coherency or inter-image alignment is just as critical as for the HUD or HMD.

A processing system for an EVS must be able to accept many input image streams (video streams), of possibly different frame rates, FOVs, and pixel counts, along with user viewing direction (helmet angle for an HMD). The processor then maps those pixels which are within the display FOV to an appropriate location on the display. The ideal mapping solution requires strict knowledge of the 3-dimensional geometry being imaged by the sensors and displays. In order to realize this ideal mapping, the geometry being imaged must be reduced to a manageable approximation. Various techniques utilizing these mapping approximations and stitching operations accurately and in a timely manner, are discussed in this paper.

In addition to common modality sensors providing wide coverage, there may be different modality sensors, such as visible, IR, and active illumination systems covering the same FOV. In this case, image fusion techniques are used to maximize the useful information content of the display. This fusion of multiple sensors is not only a function of modality; it is dependent upon sensor resolution, environmental conditions, and sensor capabilities, as well as vehicle motion.

In most implementations, sensor coverage will not extend to a full $4 \pi$ steradian sphere. Synthetic imagery on the other hand can provide $4 \pi$ steradian coverage, thus providing additional visual information, such as 3D terrain, overhead satellite imagery, and graphical symbology (e.g. Highway In the Sky), which can be subsequently fused with the regions covered by sensors, as well as providing imagery in the otherwise void regions [2]. For the system described here, the Synthetic Vision (SV) imagery is treated as just another image sensor input. This combination of sensor imagery with synthetic imagery provides situation awareness beyond what either can provide independently.

One of the advantages of an EVS system with fixed sensors as described herein, over a turret mounted sensor system, is that the available field of regard is not limited to the turret orientation. This provides the ability for each crew member to receive the various sensor images independently, supporting a number of advanced crew member interaction features. For example, it allows some crew members to view available sensor imagery in one direction, while another crew member identifies an independent area of interest. Drawing tablet (symbology) overlays would provide further graphic communication, while automatic target trackers could be launched as "virtual crew members" to track designated objects of interest.

This paper describes the image processing algorithms for stitching and fusing, and how these algorithms can be optimized to reduce latency to acceptable levels. In addition, the paper provides an overview of real-time hardware implementation considerations.

\section{ARCHITECTURE \& COORDINATE SYSTEM}

Combining imagery from multiple distributed aperture sources requires two basic mathematical components. The first component involves registration, or pixel correspondence, where pixels from the various sources and displays are geometrically correlated. The second component involves blending and fusion, where the corresponding source pixel amplitudes are combined to achieve seamless mosaics and multi-spectral imagery. The EVS system uses a central coordinate system fixed with respect to the vehicle as its primary coordinate system. The local coordinate systems for all displays and sources are referenced to this primary coordinate system. Fictitious geometric surfaces used to approximate the 3-D spatial locations of source imagery are also referenced to this primary coordinate system. Example surfaces are a sphere, used for a rough range estimate for objects of interest, and/or a plane, used as a rough estimate of the ground surface. Thus imagery from different sensors is mapped onto these geometric surfaces, as are pixels from the display device. The intersection of display pixels and sensor pixels on the surfaces represents the sensor pixels of interest (figure 2). As the user Line Of Sight (LOS) changes due to head motion, this region will change. The mapping surfaces are referenced to the primary (vehicle) coordinate system so that they follow the vehicle dynamics of translation or orientation. Imagery from the sensors should be mapped onto the surfaces so that, if they were transparent, the imagery would closely register with the real world beyond this surface, from the display perspective. 


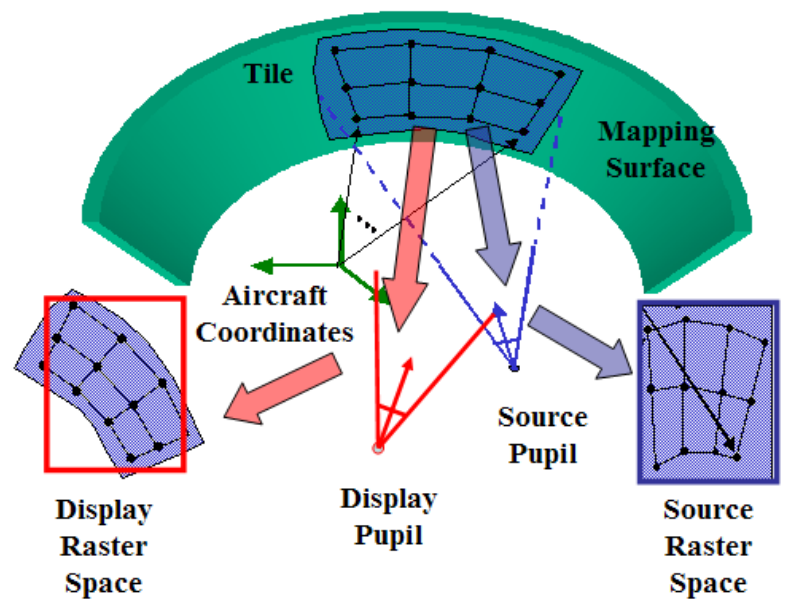

Figure 2. Primary, display, and source coordinate systems, with mapping surfaces

In general, the sensors will have solid angle Fields Of View (FOVs) such that the amount of image overlap between adjacent sensors will be range dependent (figure 3). As a result, the radius of the mapping sphere is important and should be matched to the range from the sensors to the objects being imaged. Likewise, the distance and attitude of the mapping ground plane should closely match the true ground surface. This is discussed in more detail in the next section on Pixel Correspondence. Multiple sensors operating in similar wavebands may be combined or stitched together, providing large mosaic coverage from discrete aperture sources. This mosaic combination is discussed in the section on Image Stitching. Additionally, multiple sensors operating in different wavebands may be fused together, providing multiple images for certain portions of the mapping surface. Fusion of this imagery into a single display image is discussed in the section on Sensor Fusion.
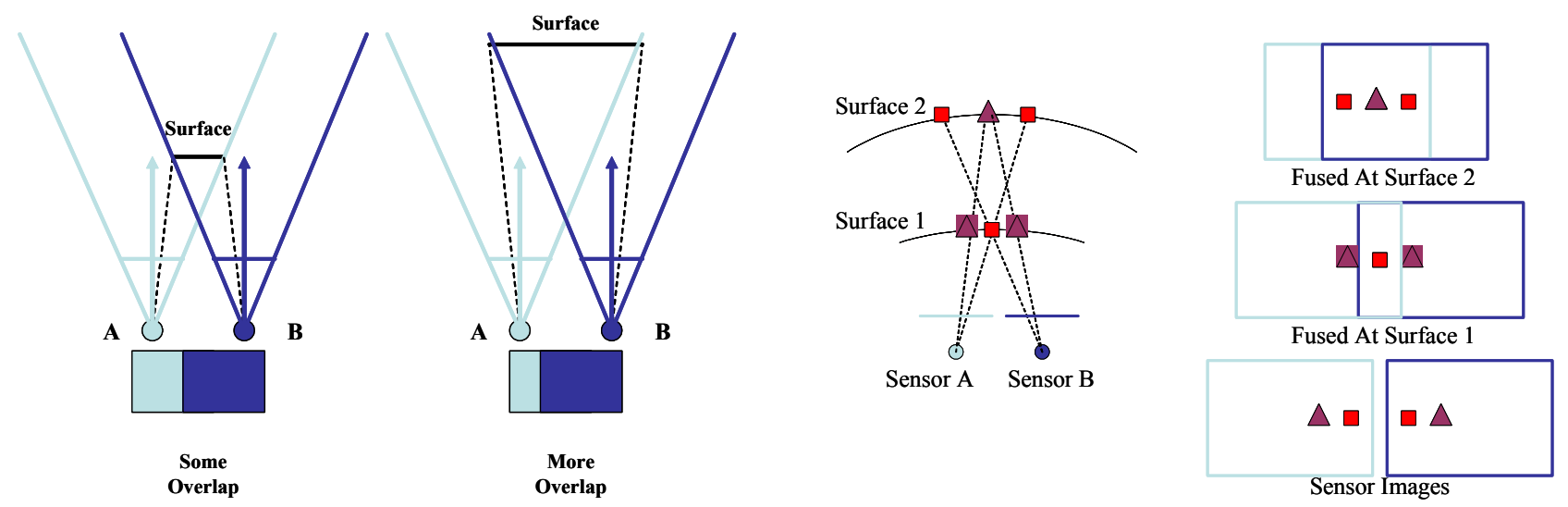

Figure 3. Ground Plane / Range Sphere Pixel Mapping Geometry

Auxiliary virtual displays may also be added as picture-in-picture or picture overlays within these areas not covered by available sensors. Pertinent examples of these auxiliary displays include a "rear view mirror" display, moving map display, own-ship displays (synthetic vision of the vehicle and surrounding terrain as seen from outside the vehicle), vehicle status displays, and crew member communication displays. Although specific examples may be presented, appropriate geometric layout of these displays is application dependent, and involves human factors issues beyond the scope of this paper. 
Potential imaging sensors include, but are not limited to visible low-light-level CCD, Infra-Red (IR, various wavebands), Milli-Meter Wave (MMW), Ground Penetrating Radar (GPR), radar or laser radar. Depending on the platform and application these sensors may provide complete coverage in azimuth $\left(360^{\circ}\right)$ or only partial coverage, some sensors types may provide multi-sensor angular broad coverage, while others provide coverage in only a specific direction (figure 4). Different sensors may operate at different frame rates, provide different image formats (pixel count, grey scale or color), and have different electrical formats (analog or digital). Other inputs to the EVS system will be vehicle position and orientation (6DOF), and user LOS (through a head tracker).

EVS display devices may be Helmet Mounted Display (HMD), Virtual Reality (VR) glasses, Heads-Up Displays (HUD), or head-down displays (HDD). The most challenging is the HMD, which require real-time head tracking and hence real-time changes in the mapping of sensor pixels to display pixels. In addition, these displays may introduce their own image distortion which must be pre-corrected in order to produce a conformal image relative to the real world. Considering physical limitations of HMD optical designs, separate corrections are typically required for each eye. Furthermore, in order to minimize visual anomalies, total system latency from photon into the sensor to photon out of the display has been specified as low as a single frame. These pixel correspondence and latency requirements impose severe constraints upon the EVS process realization.

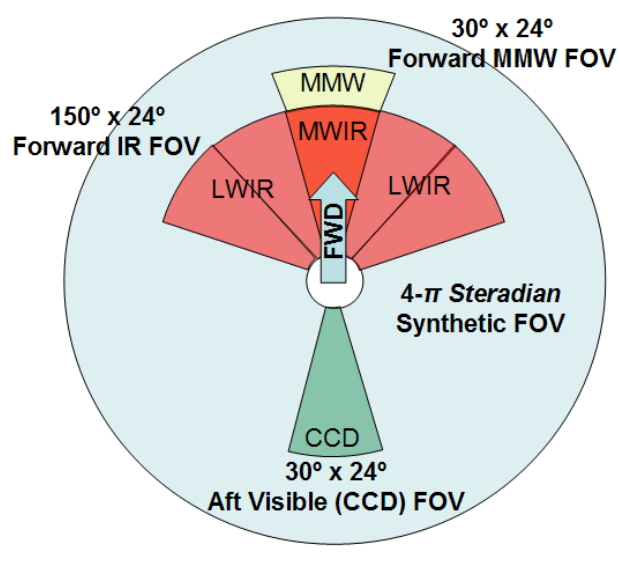

Figure 4. Example Sensor Coverage

\section{PIXEL CORRESPONDENCE}

One of the greatest challenges of implementing an effective EVS is ensuring that an object imaged by one source correctly registers in the display with the same object imaged by another source. Moreover, for conformal displays, such as an HMD or HUD, imagery in the display must correctly register with the external environment. An HMD is the most challenging case because the LOS of the display changes in real time with the operator's head movement. Since pixel correspondence for every other display type is a straightforward simplification of the HMD case, this section will focus exclusively on the HMD scenario. With this in mind, for any HMD pixel, and for every source, the pixel correspondence function determines the source pixel grid coordinates that map to the geometric center of that display pixel. Of course, some or all of the sources may not map to a particular display pixel.

The challenge of implementing an effective pixel correspondence function is twofold. Firstly, the dynamic motion of the HMD within the cockpit and of the vehicle through the environment demands that the pixel correspondence be updated in real time, which can be an enormous computational burden if many sources are used. Secondly, parallax effects for each object imaged by more than one source must be minimized so that corresponding images from separate sources correctly register on the display. These are competing objectives; either of them could be realized quite easily if the other were dismissed entirely. Achieving both simultaneously requires a realizable, but simple, strategy for each.

The first challenge of updating the pixel correspondence in real time is addressed by selecting a simple processing strategy and then finding a computational platform targeted for that strategy. The processing strategy selected was an 
independent per-display-pixel approach, or in other words, each display pixel value is calculated independently as a function of the multitude of source pixel values. This was motivated by the fact that, to the extent that the geometric approximations are valid, the source pixel correspondences can be treated independently for each display pixel. Thus, the idea was to find a single algorithm that could find the source pixel correspondences for all display pixels. However, applying such an algorithm in real time to every single display pixel could easily require that this algorithm be applied over 100 million times per second. For any nontrivial algorithm, such an overwhelming repetition rate almost necessitates that the algorithm be implemented in hardware. So, in order to accomplish a hardware implementation without sacrificing programming flexibility, it was decided to use a Field Programmable Gate Array (FPGA) for the pixel correspondence function. (The decision to also use an FPGA for the image stitching and fusion functions stemmed naturally from this.) An FPGA permits the necessary repetition rate for a sufficiently simple pixel correspondence algorithm. "Sufficiently simple," for an FPGA, means a short algorithm relying primarily on multiply-accumulate operations. Use of divisions and fractional exponentiations must be minimized, and trigonometric and other transcendental functions should be eliminated entirely, if possible. These were the essential constraints imposed on any strategy for meeting the second (parallax) challenge.

The most direct way to meet the second challenge, co-registering separate source images of the same object, is to use a range estimate to the object together with simple optics models for the sources and the display. With reasonably accurate ranges and optics models, it is a simple matter to calculate pixel correspondences. But unfortunately there may be several objects in an image with disparate ranges, and an accurate determination of these object ranges is not always feasible. However, it is possible to devise a simple scheme for determining reasonable range estimates for the most critical scenario encountered in flight - Take Off And Landing (TOAL). In a typical TOAL scenario, the aircraft is near the ground, which, one hopes, is fairly flat and relatively perpendicular to gravity. Objects along LOS vectors that ultimately intercept the ground tend to be attached to the ground in some way, or near to the ground if they are not. If they are in the near field, with LOS vector not too nearly parallel to the ground, they tend to be at a distance roughly the same as the distance to the ground along that LOS vector. If they are in the far field, with very shallow LOS vectors, the ranges are large enough that registration errors due to parallax effects are generally not a problem provided any sufficiently large range is used. For all objects somewhere in between these two extremes, a single intermediate range value can usually be used without introducing gross registration errors. Thus, one can often obtain very good registration by using a very simple geometric surface approximation. One very simple geometric surface approximation that closely matches a typical TOAL scenario, and the one that is used here, is a combined ground plane / range sphere approximation. In this approximation, a single plane is used to approximate the range to the ground and all imagery in the vicinity of the ground, and a sphere is used to approximate the range to objects in the mid-to-far field.

To accomplish the pixel correspondence, a local coordinate system is assigned and fixed with respect to each display and each source. Representing coordinate dependent vector quantities corresponding to a display or source in its respective coordinate system permits these quantities to be treated as constants in that coordinate system. For example, the LOS vector corresponding to a single HMD pixel is constant in the HMD coordinate system, even though it varies with the wearer's head motion in the primary coordinate system (fixed with respect to the aircraft). Most of the pixel correspondence calculations can be expressed in terms of these fixed quantities as a combination of rotations, translations, and scalar multiplications; calculations which are ideally suited to FPGA architecture. The calculations are further simplified by taking the origin of each display and source coordinate system to be at the optical focal point of its respective device. For a physical device, such as a sensor, the origin should correspond to the physical focal point of the device. For a virtual device, such as a synthetic vision source, the origin should correspond to the virtual viewpoint of the device. Figure 5 illustrates the concept behind the pixel correspondence algorithm.

For simplicity, consider a single pixel in a single HMD. The pixel correspondence algorithm determines the source pixel grid coordinate corresponding to the center of the display pixel for each and every source. $\mathbf{d}_{\mathbf{P}}$ is the primary coordinate representation of the vector from the origin of the primary coordinate system to the origin of the display coordinate system, and the magnitude of $\mathbf{d}_{\mathbf{P}}$ is $d$. Likewise, $\mathbf{s}_{\mathbf{P k}}$ is the primary coordinate representation of the vector from the origin of the primary coordinate system to the origin of the $k^{\text {th }}$ source coordinate system. $\mathbf{n}_{\mathbf{D}}$ is the display coordinate representation of the LOS unit vector corresponding to the HMD pixel. The set of all such vectors for every pixel in the HMD is determined by some calibration procedure, and this set remains constant provided the HMD is not altered or adjusted. The primary coordinate representation of the display pixel LOS unit vector, $\mathbf{n}_{\mathbf{P}}$, is determined by the operation 


$$
\mathbf{n}_{\mathbf{P}}=\mathbf{R}_{\mathbf{P}}^{\mathbf{D}} \mathbf{n}_{\mathbf{D}},
$$

where $\mathbf{R}_{\mathbf{P}}^{\mathbf{D}}$ is the $3 \times 3$ rotation matrix from the display coordinate system to the primary coordinate system, calculated from the HMD head tracker data.

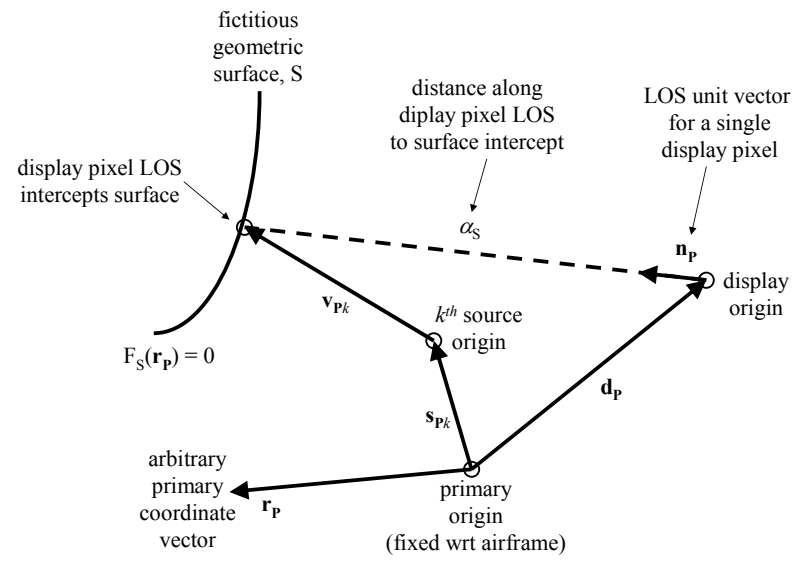

Figure 5. Conceptual Depiction of the Pixel Correspondence Algorithm

Projecting along the display pixel LOS unit vector, it may eventually intercept one of the fictitious geometric surfaces used for parallax resolution. For a display within a spherical surface, there will always be a forward and aft intercept solution. If a ground plane is present, there will be either a forward or an aft intercept solution unless $\mathbf{n}_{\mathbf{P}}$ is exactly parallel to the plane, in which case there is no solution. The distance along the LOS unit vector to the intercept with surface $\mathrm{S}$ is $\alpha_{\mathrm{S}}$. Let $\mathrm{F}_{\mathrm{S}}\left(\mathbf{r}_{\mathbf{P}}\right)=0$ be the equation for geometric surface $\mathrm{S}$, where $\mathbf{r}_{\mathbf{P}}$ is an arbitrary coordinate vector in the primary coordinate system. Then $\alpha_{\mathrm{S}}$ can be found by solving

$$
\mathrm{F}_{\mathrm{S}}\left(\mathbf{d}_{\mathbf{P}}+\alpha_{\mathrm{S}} \mathbf{n}_{\mathbf{P}}\right)=0
$$

For example, the equation for a range sphere, $\mathrm{R}$, is $\mathrm{F}_{\mathrm{R}}\left(\mathbf{r}_{\mathrm{P}}\right)=\mathbf{r}_{\mathrm{P}} \cdot \mathbf{r}_{\mathrm{P}}-R^{2}=0$, where $R$ is the radius of the sphere. Likewise, the equation for a ground plane, $\mathrm{G}$, is $\mathrm{F}_{\mathrm{G}}\left(\mathbf{r}_{\mathrm{P}}\right)=\mathbf{r}_{\mathbf{P}} \cdot \mathbf{h}_{\mathbf{P}}-H=0$, where $\mathbf{h}_{\mathbf{P}}$ is the primary coordinate representation of the normal unit vector from the primary origin to the ground plane, and $H$ is the distance along this vector from the primary origin to the ground plane. Taking the closest forward intercept along the display pixel LOS unit vector as the proper solution, the solution for $\alpha$, the distance along $\mathbf{n}_{\mathbf{P}}$ to the closest sphere/plane intercept, is given by:

$$
\begin{aligned}
& \mathrm{F}_{\mathrm{R}}\left(\mathbf{d}_{\mathbf{P}}+\alpha_{\mathrm{R}} \mathbf{n}_{\mathbf{P}}\right)=\left(\mathbf{d}_{\mathbf{P}}+\alpha_{\mathrm{R}} \mathbf{n}_{\mathbf{P}}\right) \cdot\left(\mathbf{d}_{\mathbf{P}}+\alpha_{\mathrm{R}} \mathbf{n}_{\mathbf{P}}\right)-R^{2}=\alpha_{\mathrm{R}}{ }^{2}+\left(2 \mathbf{d}_{\mathbf{P}} \cdot \mathbf{n}_{\mathbf{P}}\right) \alpha_{\mathrm{R}}+\left(d^{2}-R^{2}\right)=0 \\
& \mathrm{~F}_{\mathrm{G}}\left(\mathbf{d}_{\mathbf{P}}+\alpha_{\mathrm{G}} \mathbf{n}_{\mathbf{P}}\right)=\left(\mathbf{d}_{\mathbf{P}}+\alpha_{\mathrm{G}} \mathbf{n}_{\mathbf{P}}\right) \cdot \mathbf{h}_{\mathbf{P}}-H=\left(\mathbf{n}_{\mathbf{P}} \cdot \mathbf{h}_{\mathbf{p}}\right) \alpha_{\mathrm{G}}+\left(\mathbf{d}_{\mathbf{p}} \cdot \mathbf{h}_{\mathbf{P}}-H\right)=0 \\
& \alpha=\inf \left\{\alpha \geq 0: \alpha \in\left\{\alpha_{\mathrm{R}}, \alpha_{\mathrm{G}}\right\}\right\}
\end{aligned}
$$

With the intercept now determined, the primary coordinate representation of the vector from the origin of the $k^{\text {th }}$ source coordinate system to the display pixel LOS / geometric surface intercept is: 


$$
\mathbf{v}_{\mathbf{P} k}=\left(\mathbf{d}_{\mathbf{P}}+\alpha \mathbf{n}_{\mathbf{P}}\right)-\mathbf{s}_{\mathbf{P} k},
$$

and the $k^{\text {th }}$ source coordinate representation of this same vector is:

$$
\mathbf{v}_{\mathbf{S} k}=\mathbf{R}_{\mathbf{S} k}^{\mathbf{P}} \mathbf{v}_{\mathbf{P} k},
$$

where $\mathbf{R}_{\mathbf{S} k}^{\mathbf{P}}$ is the $3 \times 3$ rotation matrix from the primary coordinate system to the $k^{\text {th }}$ source coordinate system, which is a constant matrix if the source is fixed with respect to the airframe. $\mathbf{v}_{\mathbf{S} k}$ is the LOS vector, in the local $k^{\text {th }}$ source coordinate system, from the focal point of that source to the estimated location imaged by the display pixel. An optical model is now applied to $\mathbf{v}_{\mathbf{S} k}$ to determine where this LOS maps onto the source Focal Plane Array (FPA). Let $\mathbf{P}_{\mathbf{S} k}\left(\mathbf{v}_{\mathbf{S} k}\right)$ be the function that performs this mapping, $\mathbf{x}_{\mathbf{S} k}$ be the $k^{\text {th }}$ source coordinate representation of the unit vector in the horizontal direction of the FPA, $\mathbf{y}_{\mathbf{S} k}$ be the $k^{\text {th }}$ source coordinate representation of the unit vector in the vertical direction of the FPA, $\beta_{k}$ be the number of pixels per unit distance along the horizontal direction of the FPA, and $\gamma_{k}$ be the number of pixels per unit distance along the vertical direction of the FPA. Then $\mathrm{c}_{k}$ and $\mathrm{r}_{k}$, the $k^{\text {th }}$ source FPA column and row coordinates that correspond to the display pixel are:

$$
\begin{aligned}
& c_{k}=\beta_{k} \mathbf{x}_{\mathbf{S} k} \cdot \mathbf{P}_{\mathbf{S} k}\left(\mathbf{v}_{\mathbf{S} k}\right), \\
& r_{k}=\gamma_{k} \mathbf{y}_{\mathbf{S} k} \cdot \mathbf{P}_{\mathbf{S} k}\left(\mathbf{v}_{\mathbf{S} k}\right) .
\end{aligned}
$$

An additional offset should be added if the origin of the FPA coordinates is not chosen to coincide with the point where the source $\mathrm{z}$ axis penetrates the FPA.

For example, suppose that the $k^{\text {th }}$ source can be represented using a simple pinhole optics model. In this case, since the origin is taken as the optical focal point, the point where each source LOS vector gets mapped onto the FPA plane is the point where the line containing the LOS vector intercepts the FPA plane. In other words, $\mathbf{v}_{\mathbf{S} k}$ gets mapped to some vector $\varepsilon_{k} \mathbf{v}_{\mathbf{S} k}$, where $\varepsilon_{k}$ is a scalar. If $\mathbf{f}_{\mathbf{S} k}$ is the normal unit vector from the $k^{\text {th }}$ source origin (the focal point) to the FPA plane, and $F_{k}$ is the focal length, or perpendicular distance from the focal point to the FPA plane, then the equation of the FPA plane is $\mathbf{r}_{\mathbf{S} k} \cdot \mathbf{f}_{\mathbf{S} k}-F_{k}=0$, where $\mathbf{r}_{\mathbf{S} k}$ is an arbitrary coordinate vector in the $k^{\text {th }}$ source coordinate system. For the case where $\mathbf{r}_{\mathbf{S} k}=\varepsilon_{k} \mathbf{v}_{\mathbf{S} k}$, the solution is $\varepsilon_{k}=F_{k} /\left(\mathbf{v}_{\mathbf{S} k} \cdot \mathbf{f}_{\mathbf{S} k}\right)$, or $\mathbf{P}_{\mathbf{S} k}\left(\mathbf{v}_{\mathbf{S} k}\right)=\varepsilon_{k} \mathbf{v}_{\mathbf{S} k}=\left[F_{k} /\left(\mathbf{v}_{\mathbf{S} k} \cdot \mathbf{f}_{\mathbf{S} k}\right)\right] \mathbf{v}_{\mathbf{S} k}$. Thus, the solution for $\mathrm{c}_{k}$ is $\mathrm{c}_{k}$ $=\beta_{k} F_{k}\left(\mathbf{v}_{\mathbf{S} k} \cdot \mathbf{x}_{\mathbf{S} k}\right) /\left(\mathbf{v}_{\mathbf{S} k} \cdot \mathbf{f}_{\mathbf{S} k}\right)$, with a similar solution for $\mathbf{r}_{k}$. Since dot products are invariant under orthogonal coordinate transformations, this can also be written as $\mathrm{c}_{k}=\beta_{k} F_{k}\left(\mathbf{v}_{\mathbf{P} k} \cdot \mathbf{x}_{\mathbf{P} k}\right) /\left(\mathbf{v}_{\mathbf{P k}} \cdot \mathbf{f}_{\mathbf{P k}}\right)$, where $\mathbf{x}_{\mathbf{P} k}$ and $\mathbf{f}_{\mathbf{P} k}$ are the primary coordinate representations of $\mathbf{x}_{\mathbf{S} k}$ and $\mathbf{f}_{\mathbf{S} k}$, respectively. For sources that are fixed with respect to the airframe, $\mathbf{x}_{\mathbf{P} k}$ and $\mathbf{f}_{\mathbf{P} k}$ are constant and easily calculated in advance by applying the rotation matrix from the $k^{\text {th }}$ source coordinate system to the primary coordinate system to both $\mathbf{x}_{\mathbf{S} k}$ and $\mathbf{f}_{\mathbf{S} k}$.

For source optics that can not be represented by a simple pinhole model, two alternative approaches are possible. One is to use an accurate optics model, $\mathbf{P}_{\mathbf{S} k}\left(\mathbf{v}_{\mathbf{S} k}\right)$. Another is to use the pinhole model as a first approximation and then apply a distortion model to the result. The distortion model used will depend critically on the source optics, so it is represented abstractly here by a function $\mathbf{D}_{k}\left(\mathrm{c}_{k}, \mathrm{r}_{k}\right)$. Provided the source distortion relative to a pinhole model is not too severe, the later approach is more computationally efficient because it allows all the steps from the calculation of the source LOS vector up to the initial $1^{\text {st }}$ order estimate of the source FPA location corresponding to the display pixel to be combined into a single simple linear calculation that is followed by a single low order nonlinear correction. The algorithm is determined by taking the expression generated for $\mathrm{c}_{k}$ in the previous paragraph, substituting the expression for $\mathbf{v}_{\mathbf{P} k}$ from the above equations, and rearranging terms. Bear in mind that, provided the sources are fixed with respect to the airframe, the quantities $\left[\left(\mathbf{d}_{\mathbf{P}}-\mathbf{s}_{\mathbf{P} k}\right) \cdot \mathbf{x}_{\mathbf{P} k}\right],\left[\left(\mathbf{d}_{\mathbf{P}}-\mathbf{s}_{\mathbf{P} k}\right) \cdot \mathbf{f}_{\mathbf{P} k}\right]$, and $\left[\left(\mathbf{d}_{\mathbf{P}}-\mathbf{s}_{\mathbf{P} k}\right) \cdot \mathbf{y}_{\mathbf{P} k}\right]$ are all scalar values that only need be determined once, when the algorithm is initialized. Summarizing the final result, the pixel correspondence algorithm is: 


$$
\begin{aligned}
& \mathbf{n}_{\mathbf{P}}=\mathbf{R}_{\mathbf{P}}^{\mathbf{D}} \mathbf{n}_{\mathbf{D}} \\
& \left(H, \mathbf{h}_{\mathbf{P}}\right)=\text { (altitude, ground direction) (calculated from aircraft altitude and attitude) } \\
& R=H / \sin \theta \\
& \alpha_{\mathrm{R}}=\sqrt{\left(\mathbf{d}_{\mathbf{p}} \cdot \mathbf{n}_{\mathbf{p}}\right)^{2}+\left(R^{2}-d^{2}\right)}-\left(\mathbf{d}_{\mathbf{p}} \cdot \mathbf{n}_{\mathbf{p}}\right) \quad \text { (forward range sphere intercept) } \\
& \alpha_{\mathrm{G}}=\left(H-\mathbf{d}_{\mathbf{p}} \cdot \mathbf{h}_{\mathbf{P}}\right) /\left(\mathbf{n}_{\mathbf{p}} \cdot \mathbf{h}_{\mathbf{P}}\right) \\
& \text { (ground plane intercept) } \\
& \alpha=\inf \left\{\alpha \geq 0: \alpha \in\left\{\alpha_{\mathrm{R}}, \alpha_{\mathrm{G}}\right\}\right\} \\
& \text { (choose closest foward intercept) }
\end{aligned}
$$

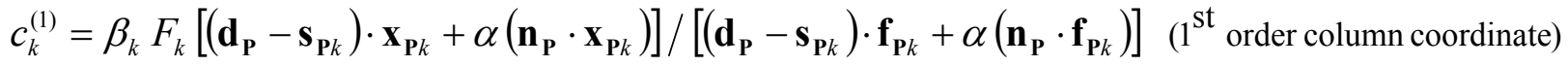

$$
\begin{aligned}
& r_{k}^{(1)}=\gamma_{k} F_{k}\left[\left(\mathbf{d}_{\mathbf{P}}-\mathbf{s}_{\mathbf{P} k}\right) \cdot \mathbf{y}_{\mathbf{P} k}+\alpha\left(\mathbf{n}_{\mathbf{P}} \cdot \mathbf{y}_{\mathbf{P} k}\right)\right] /\left[\left(\mathbf{d}_{\mathbf{P}}-\mathbf{s}_{\mathbf{P} k}\right) \cdot \mathbf{f}_{\mathbf{P} k}+\alpha\left(\mathbf{n}_{\mathbf{P}} \cdot \mathbf{f}_{\mathbf{P} k}\right)\right] \text { (1 }{ }^{\text {st }} \text { order row coordinate) } \\
& \left(\begin{array}{c}
c_{k} \\
r_{k}
\end{array}\right)=\mathbf{D}_{k}\left(c_{k}^{(1)}, r_{k}^{(1)}\right)
\end{aligned}
$$

\section{IMAGE STITCHING}

Partially overlapping imagery from sources of the same modality should be seamlessly stitched together to present the viewer with the most cogent interpretation. It is assumed that adjustment of the source intensity gain and level has been accomplished beforehand, so that any two pixels imaging the same point from two different sources of the same modality have the same intensity. If image registration on the display were perfect in this case, stitching would be a trivial operation. One could apply any normalized linear weighting to the pixels in the overlap region and the resulting display image would be the same regardless of the weighting chosen. However, registration is rarely, if ever, perfect, so it is important to blend the imagery in the overlap region in such a way as to insure that it tapers smoothly into the nonoverlap region. Thus, two corresponding source pixels in the overlap region that are near the edge of one source but well within another should be strongly weighted towards the interior pixel so there is no evident discontinuity as the boundary from the overlap region to the non-overlap region is crossed. It is assumed that the stitching need not be a function of the pixel intensity, so the stitching operation becomes a function solely of the overlap geometry.

The basic idea behind stitching is very simple, but there is a challenge that arises when implementing an independent per-display-pixel approach, as discussed in the previous section. The motivation of this approach is to implement an efficient algorithm on an FPGA or similar architecture in such a way that each display pixel is treated as independently as possible. With pipelined gate-array processing, the ideal would be to process every display pixel without reference to any of its neighbors, and this ideal should never be violated beyond nearest-neighbor references. This mandates that the algorithm not "peak" at distant display pixels to see how close a given pixel is to the edge of an overlap region. Fortunately, there is sufficient information extracted from the pixel correspondence algorithm to avoid doing so.

A two-step strategy is implemented. First, an un-normalized stitching function is defined for each source. The unnormalized stitching function for the $k^{\text {th }}$ source, $\mathrm{S}_{k}\left(c_{k}, r_{k}\right)$, is a function solely of the source coordinates determined by the pixel correspondence algorithm. The purpose of this function is to determine the relative weighting of pixels stitched from multiple sources, and the weighting is normalized after the fact. The function should have the property that it is large when the coordinates $\left(c_{k}, r_{k}\right)$ are near the center of the FPA, approaches zero as these coordinates approach the edge of the FPA, and remains zero beyond the edge of the FPA. The un-normalized stitching function used here is:

$$
\mathrm{S}_{k}\left(c_{k}, r_{k}\right)=\left(1-\frac{2\left|c_{k}-c_{o k}\right|}{N_{x k}}\right)\left(1-\frac{2\left|r_{k}-r_{o k}\right|}{N_{y k}}\right) H\left(1-\frac{2\left|c_{k}-c_{o k}\right|}{N_{x k}}\right) H\left(1-\frac{2\left|r_{k}-r_{o k}\right|}{N_{y k}}\right)
$$


where $\left(c_{o k}, r_{o k}\right)$ are the coordinates of the geometric center of the $k^{t h}$ FPA, $N_{x k}$ and $N_{y k}$ are the number of pixels along its horizontal and vertical directions, respectively, and $H(x)$ is the Heaviside step function. The stitching function applies to stitched sources, not fused sources. A contour plot of this function is shown if Figure 6.

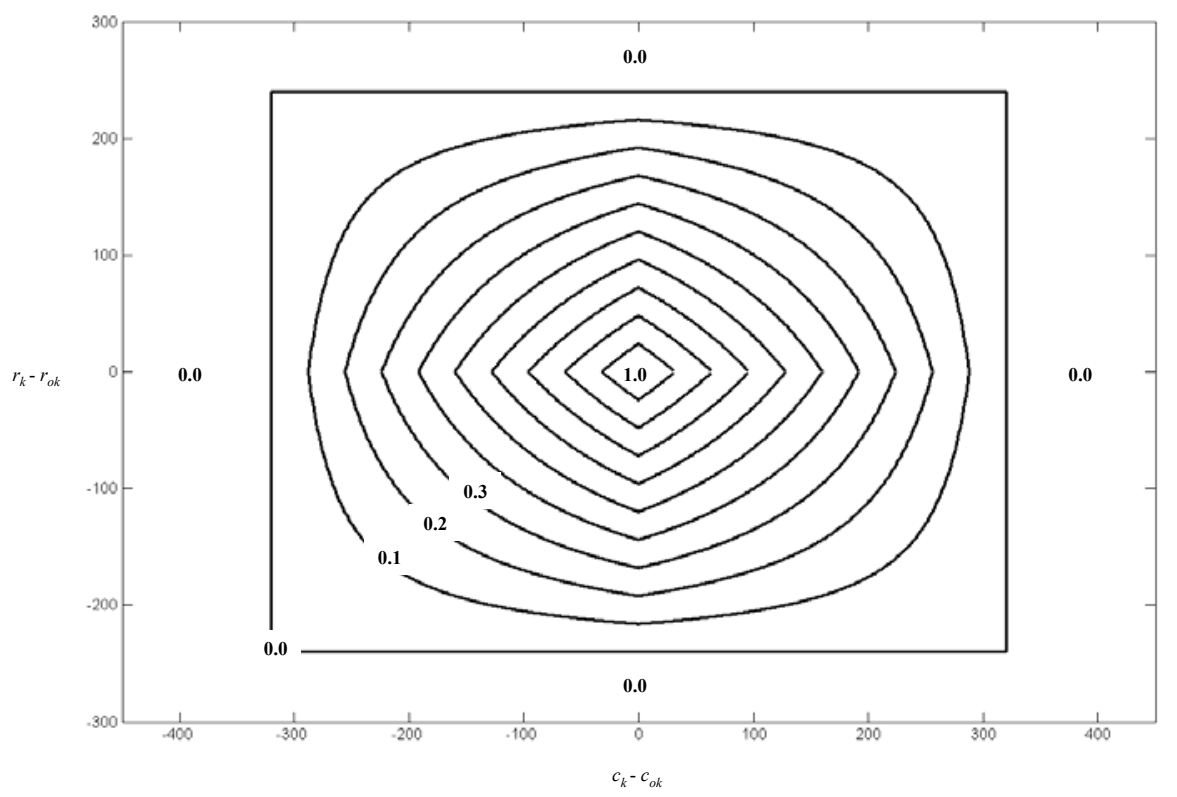

Figure 6. Contour Plot of the Un-normalized Stitching Function for a 640 x 480 FPA

The stitching blending coefficient for the $k^{\text {th }}$ source at a given display pixel is determined by normalizing all the unnormalized stitching values for that display pixel:

$$
b_{k}=\frac{\mathrm{S}_{k}\left(c_{k}, r_{k}\right)}{\sum_{\substack{n \\ \text { stitched }}} \mathrm{S}_{n}\left(c_{n}, r_{n}\right)} .
$$

The total stitched intensity for that display pixel is then:

$$
I_{\text {stitched }}=\sum_{\substack{k \\ \text { stitched }}} b_{k} I_{k}\left(c_{k}, r_{k}\right),
$$

where $I_{k}\left(c_{k}, r_{k}\right)$ is the $k^{\text {th }}$ source intensity value at $\left(c_{k}, r_{k}\right) . I_{k}\left(c_{k}, r_{k}\right)$ can be determined by nearest-neighbor interpolation, bilinear interpolation, or whatever means is most appropriate for the application at hand.

The advantage of the above approach is its simplicity and that it does not rely on non-local display pixel data, which may not have yet been generated. Also, it automatically results in the most intuitive result for the common case of two adjacent sources of the same resolution whose top and bottom, or left and right, edges are aligned. As is easily confirmed 
by direct calculation using the above equations, the blending profile in this case is a linear ramp in the overlap region. This is illustrated in Figure 7.

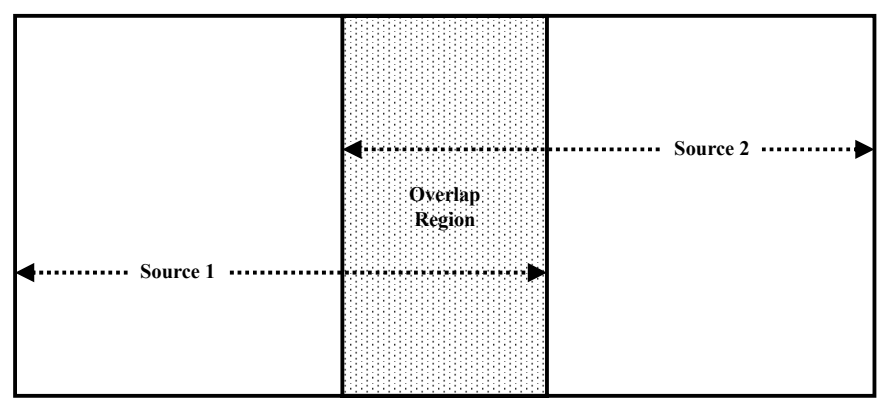

Identical \& Aligned Adjacent Sources

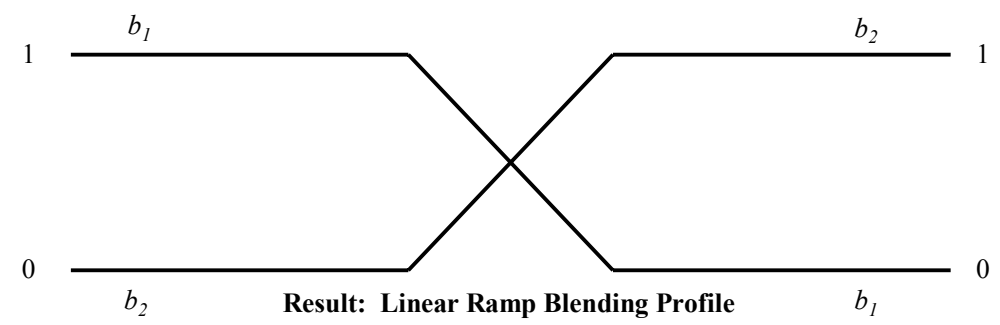

Figure 7. Blending Profile Generated by the Stitching Algorithm for the Common Case of Two Identical Adjacent Sources with Top and Bottom Edges Aligned

Testing shows that this stitching procedure produces visually pleasing results even for sources overlapping in a skewed fashion, though the blending profiles are not strictly linear in these cases. Also, picture-in-picture imagery not overlapping with other sources is automatically placed in a window with a unit-blending footprint by this approach. An example of combined stitching and blending is shown in figure 8.

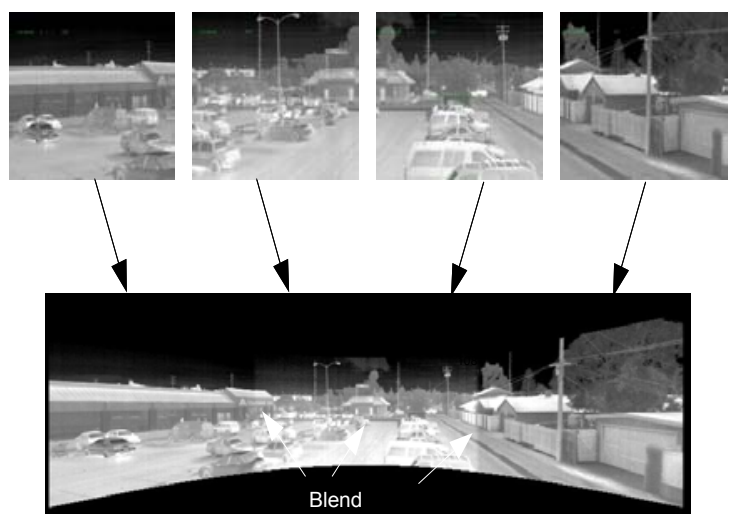

Figure 8. Image stitching and blending

\section{IMAGE FUSION}

The underlying premise of an Enhanced Vision System is to provide the vehicle navigator with maximal situational awareness with minimal interpretation. The obvious solution is to combine information content from each and every 
available imaging sensor into some composite image that presents this maximal, easily interpretable image. In order to combine images from discrete (distributed aperture) sources, the issue of pixel correspondence must first be addressed, as expressed in previous sections of this paper. Once this registration is achieved, the remaining problem involves some mathematical combination of the corresponding pixel amplitudes to achieve the desired resultant image.

Industry and academia efforts over the past few years have shown that combination of multi-spectral imagery can be either beneficial or detrimental, depending upon which source provides what information at which time [3-12]. In order to maximize benefit from the available information, a statistical relationship between the image sources must be derived such that the corresponding pixel amplitudes may be combined by some desired mathematical principal. Taking into account the implications from academia and industry, the ultimate image fusion algorithm will most likely elude mankind for some time. Meanwhile, there seem to be a few approximations that may provide an acceptable short-term solution.

Simply blending images together on a percentage basis can provide a first-order approximation to the image fusion problem (figure 9). This can be exemplified by a person standing in a dark doorway. He cannot be seen in the visible spectrum, but stands out like a sore thumb in the long-wave infrared spectrum. Simply adding these two images together provides a compelling result. Unfortunately, this approach has many shortfalls. For instance, if the walls behind the person standing in the dark doorway were at 98.6 degrees Fahrenheit, the only discerning factor would be clothing.

Moving beyond the simple linear combination of images requires some statistical measurement of image content, contrast, quality, or metric-du-jour, in order to determine which portions of what image can provide significant information. One approach is to apply global spatial analysis, typically the frequency spectrum, where significant information may be extracted and then recombined by the inverse conversion. Unfortunately, conversion to and from global spaces typically requires image frame buffering, and thus tend to exceed HMD and even HUD latency requirements.

An alternative to global spatial analysis involves local content analysis, where each contributing source pixel is weighted according to the results of some convolution or other mathematical relationship of neighboring source pixels. This approach has obvious latency advantages due to its localized nature. The inherent parallel processing architecture that may be applied in this case provides a significant advantage over the global spatial approach. Given today's gate array processors, these localized algorithms can be realized on a very large scale, supporting large numbers of high-resolution image sources, as well as high-resolution display outputs.

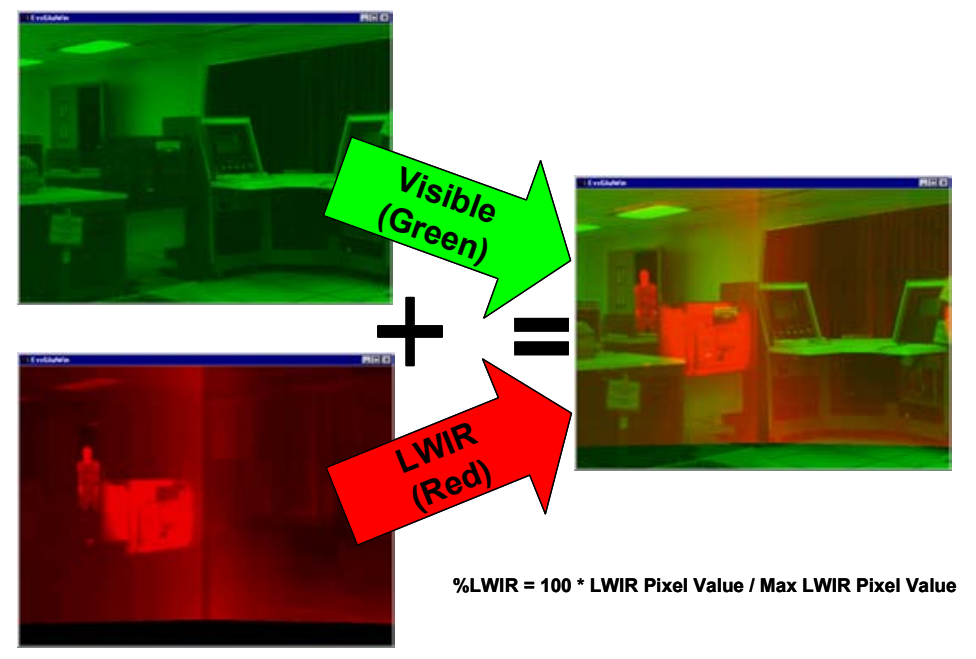

Figure 9. Simple image fusion algorithm

Formally, both blending and local content analysis can be applied in a manner similar to that of stitching. In this case, the stitching function for the $k^{\text {th }}$ source, $\mathrm{S}_{k}\left(c_{k}, r_{k}\right)$, is replaced with a corresponding fusion function, $\mathrm{F}_{k}\left(c_{k}, r_{k}\right)$. Likewise, 
the stitching blending coefficient, $b_{k}$, is replaced with a corresponding fusion coefficient, $f_{k}$. The essence of a fusion algorithm is how $\mathrm{F}_{k}\left(c_{k}, r_{k}\right)$ and $f_{k}$ are calculated. For efficient gate array processing, these calculations should exclusively involve a few local arithmetic operations, primarily addition and multiplication. Normalization divisions, such as the one in equation (12), are acceptable because these involve taking a single reciprocal (division) and multiplying several unnormalized quantities by this value. To limit raster scan delays, neighborhood dependencies should be limited to nearest neighbors. Once the fusion coefficients are determined, the fusion intensities are calculated by:

$$
I_{\text {fused }}=\sum_{\substack{k \\ \text { fused }}} f_{k} I_{k}\left(c_{k}, r_{k}\right),
$$

where the summation is over fused sources only.

In addition to the parallel processing advantage, the gate array implementation provides today's EVS efforts with the almost mandatory ability to modify or program the underlying fusion algorithms. The need for this programmability is evident in the sheer number of engineers and scientists studying the problem throughout the world today, and the fact that there is no one fusion solution available to date that can boast the robustness demanded by the vehicle navigators expected to trust EVS imagery with their lives and those of their passengers.

\section{SYNTHETIC VISION}

The easiest way to introduce Synthetic Vision (SV) is to describe the typical flight simulator program with which almost any computer enthusiast is familiar. With SV, the viewpoint within some virtual terrain database is correlated with the vehicle and display kinematics such that the vehicle navigator sees imagery conformal with the real world. There are several obvious pitfalls with this approach, involving errors in vehicle geometry, display geometry, and yes, believe it or not, database geometry errors. As with the image fusion community, there is a large contingent of academia and industry that is addressing database accuracy issues, which are beyond the scope of this paper. The concepts of SV within this paper should be revered as somewhat ideal, with the caveat that the reality of error is understood when it is not directly expressed.

Considering an EVS processor that combines multiple image sources to increase situational awareness of a vehicle navigator, the advantages of SV sources are quite obvious. Although SV can provide compelling, almost perfect imagery, it is always subject to the trust of the vehicle navigator that can be easily swayed by errors in vehicle kinematics and database geometry. For instance, given vehicle location errors, the SV will obviously provide inaccurate information, where the generated imagery is no longer conformal with the real world. This undesirable result competes with the issue of database errors as the primary reason why SV cannot be trusted alone. However, fusing the SV with sensor imagery can provide verification between the synthetic and the real world, as well as increased information content when they are accurately aligned. This combination of SV and sensor imagery can significantly increase the level of trust, and is rapidly becoming the number-one desired capability within the EVS community.

The mathematics of pixel correspondence for SV sources is exactly the same as discussed above, except that the source parameters (position, attitude, FOV, resolution, etc.) are all virtual, and there is no need for distortion correction. Likewise, SV fusion can be handled in the manner discussed in the "Image Fusion" section (stitching is a non-issue since SV imagery is generally not stitched).

In addition to fusion of sensor imagery with the 3D SV terrain, graphical symbology overlays can provide unprecedented situational awareness. These overlays combine vehicle state information, as provided by typical head-up displays, with advanced flight guidance symbology, such as geo-specific waypoints, highway-in-the-sky, threat depiction, etc (figure 10). It is important to note that the fusion process between sensor and SV sources should involve only the terrain portion of the SV, and that the symbology should be implemented as an overlay. Although a simple concept, this separation of terrain and symbology proves to be detrimental in the gate array implementation, where the SV is required to be split into two independent image sources, further complicating the video input hardware design. Fortunately, this is not a fatal shortcoming; it simply adds to the processing overhead. 

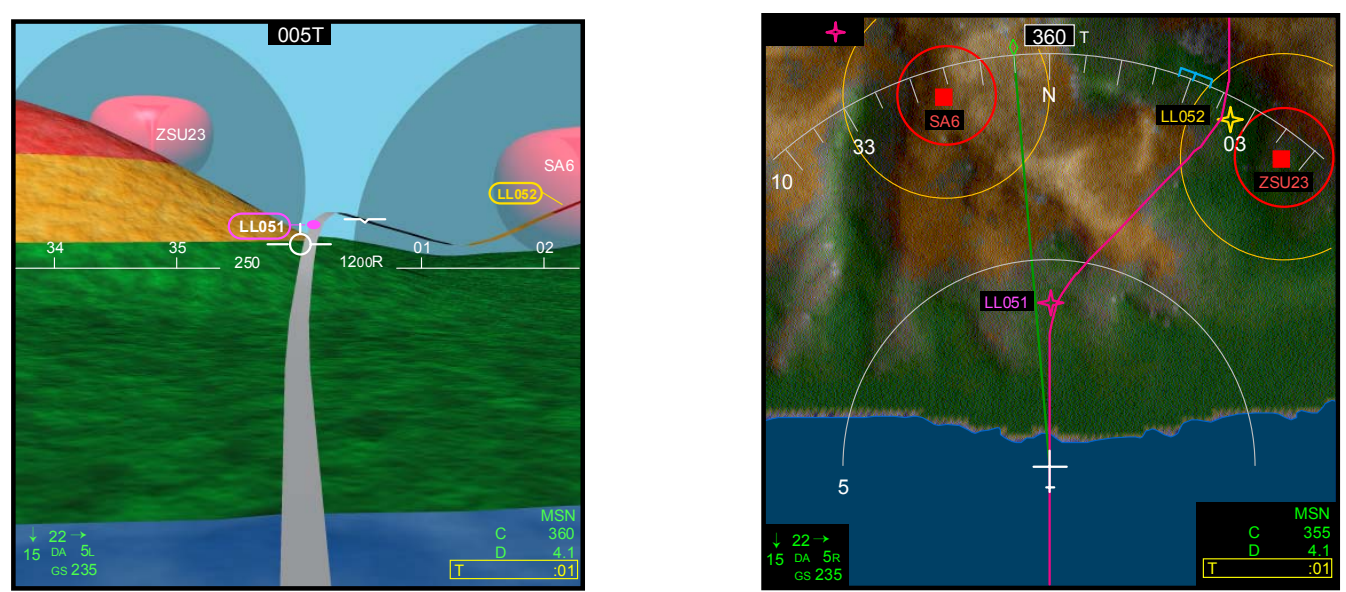

Fig 10. Left: Synthetic Imagery containing digital elevation maps, overhead imagery, and flight symbology. Right: Moving Map display.

Probably the most compelling attribute of SV is the $4 \pi$ steradian field of regard advantage of head-mounted displays. Given accurate and low-latency measurement of head orientation, the SV provides conformal imagery by combining head and vehicle kinematics relative to the geo-specific database elements. This provides a clear advantage to the headmounted display, versus the fixed-forward view of the head-up or head-down displays. Thus for the HMD, the headtracked SV can then be fused with the various vehicle-stable sensors by considering the head motion as a deviation to the nominal vehicle-stable display coordinate system during the Pixel Correspondence phase of the EVS algorithm.

\section{IMPLEMENTATION}

In order to demonstrate the concepts discussed in this paper, Boeing has performed a number of development and demonstration efforts. Previous efforts include the use of a rack mounted PC capable of handling a limited number of low resolution $(640 \times 480)$ sensors. This system was developed using the OpenGL toolkit and resulted in both a flight demonstration and a test van used to evaluate sensor combinations and integration [1].

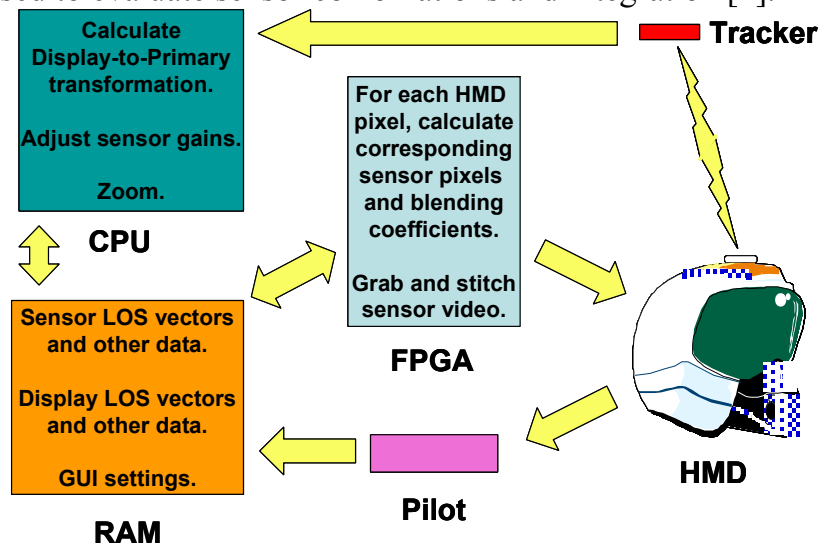

Figure 11. EVS block diagram

As the technology has matured, it was decided to proceed with a high performance hardware implementation. The requirements for this system were to support up to $8,1 \mathrm{~K} \mathrm{x} 1 \mathrm{~K}, 60 \mathrm{~Hz}$ (progressive scan) sensors, synthetic vision input, HMDs with separate left and right eye warping, and single frame processing latency from photon into a sensor to photon out of the display. Some of these sensors could be partially overlapping common modality sensors, while others could be different modality sensors requiring image fusion. In order to achieve this performance level, a custom board design has been fabricated using FPGA logic chips. The general architecture is shown in figure 11. 
In the current system, there is one EVS processor card per user (HMD). Additional users can be supported with the same set of sensors by simply adding additional displays and processor cards.

\section{SUMMARY}

The requirements of an Enhanced Vision System resulting from a large number of high resolution, high frame rate sensors and low latency requirements, result in stressing demands on both algorithms and processors. FPGA processing technology is well suited to image processing applications such as EVS in which simple operations are performed on large numbers of pixels. However, even with the parallel processing power available from current FPGA hardware, algorithmic challenges persist. We have described the sensor to display pixel mapping, the image stitching and fusing algorithms. Each of the transformations in the image processing pipeline can be thought of as a matrix operation on the incoming pixel arrays. By examining the sequence of operations, we have been able to combine what was formerly a sequential set of operations into just a few matrix operations which can be efficiently implemented.

The result is a high performance system providing wide area live coverage, high resolution, low latency, multiple modality sensor fusion, and integrated synthetic vision for situational awareness. The current system is highly scalable to additional users. The current system is a "dumb" system in the sense that it provides integrated imagery to a human user who supplies all of the intelligence for interpretation. However, there is no reason that in the future, some of the users could be "intelligent agents" of various sorts.

\section{REFERENCES}

1. J. Guell, "Flying Infrared for Low-level Operation (FLILO) Systems Description and Capabilities", Proc $18^{\text {th }}$ Digital Avionics Systems Conference, 1998.

2. C.W. Jennings, K.W. Alter, A.K. Barrows, K.L. Bernier, J.J. Guell, "Synthetic Vision as an Integrated Element of an Enhanced Vision System”, Proc SPIE, 4713, April, 2002.

3. P.J. Burt, R.J. Kolczynski; "Enhanced image capture through fusion", Proc $4^{\text {th }}$ International Conf on Computer Vision, 1993. pp 173-182.

4. W.K. Krebs, D.A. Scribner, G.M. Miller, J.S. Ogawa, J. Schuler; "Beyond Third Generation: A sensor fusion targeting FLIR pos for the F/A-18", Proc of the SPIE, 3376, 1998. pp 129-140.

5. H. Li, B.S. Manjunath, S.K. Mitra; "Multisensor image fusion using the wavelet transform", Graphical Models and Image Processing, 57 (3), 1995. pp 235-245.

6. C. Perneel; "Multi-level fusion for the detection of targets using multi-spectral image sequences", Opt Eng, 37 (2), 1998. pp 477-484.

7. R.K. Sharma, M. Pavel, T.K. Leen; "Multi-stream video fusion using local principal components analysis", Proc SPIE, 3436, 1998.

8. R.K. Sharma, T.K. Leen, M. Pavel; "Probabilistic Image Sensor Fusion", Advances in Neural Information Processing Systems, The MIT Press, 1999.

9. A. Toet; "Hierarchical Image Fusion", Machine Vision and Applications, 3, 1990. pp 1-11.

10. A.T. Wilson, K.S. Rogers, R.L. Myers; "Perceptual-based hyperspectral image fusion using multiresolution analysis", Opt Eng, 34 (11), 1995. pp 3154-3164.

11. P. Simard, N.K. Link, R.V. Kruk; "Feature detection performance with fused synthetic and sensor images", Proc Human Factor and Ergonomics Society, 43 ${ }^{\text {rd }}$ Annual Meeting, Houston, TX. pp 11081112.

12. A. Toet, J.K. Ijspeert, A.M. Waxman, M. Aguilar; "Fusion of visible and thermal imagery improces situational awareness", Displays, 18, 1997. pp 85-95. 